\title{
Origin of anomalies in the path-integral formalism
}

\author{
Izumi Tsutsui \\ II. Institut für Theoretische Physik der Universität Hamburg, D-2000 Hamburg 50, Federal Republic of Germany
}

(Received 31 May 1989)

\begin{abstract}
We show that, in the path-integral formalism, anomalies can arise from the discrepancy between classical field equations and quantum field equations. With a suitable regularization for the functional derivative, this discrepancy leads to an expression identical to that obtained from Fujikawa's anomalous Jacobian, for the $\mathrm{U}(1)$ and the non-Abelian anomalies, respectively. This approach provides an alternative interpretation for the origin of anomalies in the path-integral formalism, which is more closely related to the conventional one in the operator formalism.
\end{abstract}

\section{INTRODUCTION}

A decade ago, Fujikawa ${ }^{1}$ studied in a series of papers the origin of anomalies in the path-integral formalism. There he revealed that anomalies are caused by the Jacobian factor, which arises from the noninvariance of the path-integral measure under infinitesimal transformations. He also evaluated the anomalous Jacobian and related it to the local version of Atiyah-Singer index theorem. Since then, his approach has been studied extensively, and has become a standard method valid for all the known anomalies related to infinitesimal transformations. ${ }^{2}$

In this paper we show that there is an alternative interpretation for the origin of anomalies in the path-integral formalism, which is more closely related to the one in the operator formalism: namely, the discrepancy between classical field equations and quantum field equations. In classical theories, (classical) field equations are derived from the variational principle, i.e., the variation of the action $\delta I / \delta \phi$ should vanish. On the other hand, in the path-integral formalism of quantum theories, (quantum) field equations are derived from the fact that the functional integral of functional derivative vanishes identical$1 y .^{3}$ This guarantees that $\langle\delta I / \delta \phi\rangle$ vanishes also in the path integral. However, when this is multiplied by a functional $F[\phi]$ to be a composite form as $\langle F[\phi] \delta I / \delta \phi\rangle$, it is not clear whether or not it vanishes. These field equations are crucial to conserve the Noether current in classical theories. Thus, if they do not hold in the path integral, the current is no longer conserved and anomalies may arise. This interpretation of anomalies has also been suggested by Fujikawa, ${ }^{2}$ and Joglekar and Misra. ${ }^{4}$

From this point of view, we examine Noether's argument in the path-integral formalism, and acquire anomalies directly as a breakdown of the argument for the current conservation associated with global symmetries. Then we illustrate it by the case of the $U(1)$ and the non-Abelian anomalies. With a suitable regularization for the functional derivative, we derive an expression identical to that obtained for the anomalous Jacobian, for both anomalies, respectively. It is shown that this is not an accident but, on the contrary, Fujikawa's anomalous Jacobian is a different manifestation of the discrepancy between classical and quantum field equations. We also briefly discuss how the origin of anomalies in the pathintegral formalism is understood in the operator formalism.

\section{BREAKDOWN OF NOETHER'S ARGUMENT AND ANOMALIES}

To see why Noether's argument in classical theories may break down when one goes over to quantum theories, we will examine it in the path-integral formalism. Let $\mathcal{L}[\phi]$ be a Lagrangian for a generic field $\phi$, which is invariant under a global infinitesimal transformation $\phi \rightarrow \phi^{\prime}=\phi+\bar{\delta} \phi$. Then, for the path integral, we have

$$
\begin{aligned}
0=\langle\bar{\delta} \mathcal{L}\rangle=\frac{1}{N} \int d \phi \bar{\delta} \mathcal{L} e^{i I[\phi]} & =\frac{1}{N} \int d \phi\left\{\left[\frac{\partial \mathcal{L}}{\partial \phi}-\partial_{\mu}\left(\frac{\partial \mathcal{L}}{\partial \partial_{\mu} \phi}\right)\right] \bar{\delta} \phi+\partial_{\mu}\left(\frac{\partial \mathcal{L}}{\partial \partial_{\mu} \phi} \bar{\delta} \phi\right)\right\} e^{i I[\phi]} \\
& =\left\langle\frac{\delta I}{\delta \phi} \bar{\delta} \phi\right\rangle+\left\langle\partial_{\mu} j^{\mu}\right\rangle \alpha
\end{aligned}
$$

where $I=\int d x \mathcal{L}, N=\int d \phi e^{i I[\phi]}$ is a normalization factor, and $j^{\mu}$ is the Noether current. Here we consider a linear transformation $\bar{\delta} \phi \propto \alpha \phi$ with an infinitesimal constant parameter $\alpha$. (Euclidean prescription is always assumed for the path integral, although it is not explicitly stated.) If one is allowed to exploit the classical field equation $\delta I / \delta \phi=0$ in (1), then the Noether current is conserved. However, this is not allowed in quantum theories, because we have a slightly different quantum field equation:

$0=\frac{1}{N} \int d \phi \frac{\delta}{\delta \phi}\left(\bar{\delta} \phi e^{i I[\phi]}\right)=\left\langle\frac{\delta(\bar{\delta} \phi)}{\delta \phi}\right\rangle+i\left\langle\frac{\delta I}{\delta \phi} \bar{\delta} \phi\right\rangle$.

This gives

(c) 1989 The American Physical Society 


$$
\left\langle\partial_{\mu} j^{\mu}\right\rangle=\frac{-i}{\alpha}\left\langle\frac{\delta(\bar{\delta} \phi)}{\delta \phi}\right\rangle .
$$

Although the right-hand side (RHS) of (3) contains an ill-defined divergence $\delta(0)$, which implies that the above manipulations are formal, this relation indicates that we cannot take for granted that the Noether current is conserved in the path-integral formalism.

To be specific, we will consider the Yang-Mills theory governed by the Lagrangian

$$
\mathcal{L}=\frac{1}{2} \operatorname{tr} F_{\mu \nu} F^{\mu \nu}+\bar{\psi} i \not D \psi,
$$

where $\not D=\not \supset+A$. Under the infinitesimal global axial $\mathrm{U}(1)$ transformation,

$$
\bar{\delta} \psi(x)=i \alpha \gamma^{5} \psi(x), \quad \bar{\delta} \bar{\psi}(x)=\bar{\psi}(x) i \alpha \gamma^{5},
$$

the Lagrangian $\mathcal{L}$ in (4) is invariant. To evaluate the fermionic path integral, we will expand $\psi$ and $\bar{\psi}$ in terms of eigenfunctions $\phi_{n}$ of $i \not D$ as

$$
\psi(x)=\sum_{n} a_{n} \phi_{n}(x), \quad \bar{\psi}(x)=\sum_{n} \bar{b}_{n} \phi_{n}^{\dagger}(x),
$$

where the $\phi_{n}(x)$ 's satisfy

$$
\begin{aligned}
& i \not D \phi_{n}(x)=\lambda_{n} \phi_{n}(x), \quad \int d x \phi_{n}^{\dagger}(x) \phi_{m}(x)=\delta_{n m}, \\
& \sum_{n} \phi_{n}^{\dagger}(x) \phi_{n}(y)=\delta(x-y) .
\end{aligned}
$$

Formally, the fermionic measure is defined as $d \psi d \bar{\psi}=\Pi_{n} d a_{n} d \bar{b}_{n}$, which yields det $i \not D=\Pi_{n} \lambda_{n}$. Then the path integral may be given meaning in terms of, for instance, the $\zeta$-function regularization. We also define a regularized functional derivative as

$$
\begin{aligned}
& \frac{\delta}{\delta \psi(x)}=\lim _{M \rightarrow \infty} \sum_{n} e^{-\lambda_{n}^{2} / M^{2}} \phi_{n}^{\dagger}(x) \frac{\partial}{\partial a_{n}}, \\
& \frac{\delta}{\delta \bar{\psi}(x)}=\lim _{M \rightarrow \infty} \sum_{n} e^{-\lambda_{n}^{2} / M^{2}} \phi_{n}(x) \frac{\partial}{\partial \bar{b}_{n}} .
\end{aligned}
$$

One can check that (8) leads to the usual functional derivative, $\delta \psi(y) / \delta \psi(x)=\delta \bar{\psi}(y) / \delta \bar{\psi}(x)=\delta(x-y)$, in the limit $M \rightarrow \infty$. Then, from (3), the divergence of the axial-vector current $j_{5}^{\mu}=\bar{\psi} \gamma^{\mu} \gamma^{5} \psi$ reads

$$
\begin{aligned}
\left\langle\partial_{\mu} j_{5}^{\mu}(x)\right\rangle & =\frac{-i}{\alpha}\left[\left\langle\frac{\delta(\bar{\delta} \bar{\psi}(x))}{\delta \bar{\psi}(x)}\right\rangle+\left\langle\frac{\delta(\bar{\delta} \psi(x))}{\delta \psi(x)}\right\rangle\right] \\
& =2 \lim _{M \rightarrow \infty} \operatorname{tr} \sum_{n} \phi_{n}^{\dagger}(x) \gamma^{5} \phi_{n}(x) e^{-\lambda_{n}^{2} / M^{2}} .
\end{aligned}
$$

Equation (9) is an expression identical to that obtained by Fujikawa for the regularized anomalous Jacobian, ${ }^{1}$ which gives the axial $\mathrm{U}(1)$ anomaly

$$
\left\langle\partial_{\mu} j_{5}^{\mu}(x)\right\rangle=\frac{1}{16 \pi^{2}} \epsilon^{\mu v \alpha \beta_{\operatorname{tr}} F_{\mu \nu} F_{\alpha \beta}(x) .}
$$

For the non-Abelian anomaly, we will consider a chiral gauge theory where the gauge field couples only to a lefthanded fermion $\frac{1}{2}\left(1-\gamma^{5}\right) \psi$. Then, the Lagrangian is given by (4) with $\not D=\not+A \frac{1}{2}\left(1-\gamma^{5}\right)$, and is invariant under the following infinitesimal global chiral transforma- tion (with parameter $\alpha=\alpha^{a} T^{a}$ ):

$\bar{\delta} \psi(x)=-\alpha \frac{1}{2}\left(1-\gamma^{5}\right) \psi(x), \quad \bar{\delta} \bar{\psi}(x)=\bar{\psi}(x) \frac{1}{2}\left(1+\gamma^{5}\right) \alpha$,

$\bar{\delta} A_{\mu}^{a}(x)=\left[A_{\mu}(x), \alpha\right]^{a}$.

Proceeding in accordance with (1), we have

$$
\begin{aligned}
0 & =\langle\bar{\delta} \mathcal{L}\rangle \\
& =-\left\langle\frac{\delta I}{\delta \psi} \bar{\delta} \psi\right\rangle+\left\langle\bar{\delta} \bar{\psi} \frac{\delta I}{\delta \bar{\psi}}\right\rangle+\left\langle\frac{\delta I}{\delta A_{\mu}^{a}} \bar{\delta} a_{\mu}^{a}\right\rangle+\left\langle\partial_{\mu} j_{a}^{\mu}\right\rangle \alpha^{a}
\end{aligned}
$$

where $j_{a}^{\mu}$ is the Noether current given by

$$
\begin{aligned}
j_{a}^{\mu} & =-i \bar{\psi} T^{a} \gamma^{\mu} \frac{1}{2}\left(1-\gamma^{5}\right) \psi+\left[A_{v}, F^{\mu \nu}\right]_{a} \\
& =J_{a}^{\mu}+\left[A_{v}, F^{\mu \nu}\right]_{a} .
\end{aligned}
$$

Since the last two terms in (12) can be combined to give $\left\langle D_{\mu} J_{a}^{\mu}\right\rangle \alpha^{a}$, one ends up with

$$
\begin{aligned}
\left\langle D_{\mu} J_{a}^{\mu}(x)\right\rangle= & i \lim _{M \rightarrow \infty} \operatorname{tr} \sum_{n} \phi_{n}^{\dagger}(x) T^{a} \gamma^{5} \phi_{n}(x) e^{-\lambda_{n}^{2} / M^{2}} \\
= & \frac{i}{24 \pi^{2}} \epsilon^{\mu v \alpha \beta} \operatorname{tr} T^{a} \partial_{\mu}\left(A_{v} \partial_{\alpha} A_{\beta}\right. \\
& \left.\quad+\frac{1}{2} A_{v} A_{\alpha} A_{\beta}\right)(x),
\end{aligned}
$$

a result analogous to (9). [We note that (14) gives the consistent anomaly that is not covariant, because we employ eigenvalues $\lambda_{n}$ of the gauge-noninvariant operator $i \not D$ in (8) for this case. $\left.{ }^{5}\right]$

\section{THE ORIGIN OF ANOMALIES}

Our results, (9) and (14), strongly suggest that the origin of anomalies, i.e., the RHS of (3), may be equivalent to Fujikawa's anomalous Jacobian in general. Now we show that this is indeed the case.

If we consider the change of variables in the vacuum functional, we have

$$
\begin{aligned}
0 & =\frac{1}{N} \int d \phi^{\prime} e^{i I\left[\phi^{\prime}\right]}-\frac{1}{N} \int d \phi e^{i I[\phi]} \\
& =\frac{1}{N} \int d \phi e^{i I\left[\phi^{\prime}\right]}+\ln J-\frac{1}{N} \int d \phi e^{i I[\phi]} \\
& =\frac{1}{N} \int d \phi i \int d y\left[\frac{\delta I}{\delta \phi} \bar{\delta} \phi+\partial_{\mu}\left(j^{\mu} \alpha\right)\right] e^{i I[\phi]}+\ln J \\
& =i \int d y\left[\left\langle\frac{\delta I}{\delta \phi} \bar{\delta} \phi\right\rangle+\left\langle\partial_{\mu}\left(j^{\mu} \alpha\right)\right\rangle\right]+\ln J,
\end{aligned}
$$

where $\ln J$ represents Fujikawa's anomalous Jacobian which arises from the noninvariance of the path-integral measure. We note that (15) holds for an arbitrary infinitesimal parameter $\alpha$ which may be local. Thus, when we localize $\alpha$ at $x$ as $\alpha \rightarrow \epsilon \delta(x-y)$, we get

$$
i\left\langle\frac{\delta I}{\delta \phi} \bar{\delta} \phi\right\rangle+\left.\ln J\right|_{\operatorname{loc} \alpha}=0,
$$

since the total derivative term in (15) vanishes under the 
space-time integration. ${ }^{6}$ [Notice that this term does not necessarily vanish for any $\alpha$, because it contains topological information. It yields, for instance, the index for the Dirac operator after integration with constant $\alpha$ in the case of the U(1) anomaly.] Since it is $\ln J$ with the localized limit in (16) that gives the anomaly, ${ }^{1}$ the result (16), with (2), establishes the expected equivalence. Furthermore, by choosing a global $\alpha$ in (15), we recover the relation (1), which follows from the fact that $\ln J$ vanishes for constant $\alpha$ due to the consistency required in evaluating the anomalous Jacobian.

This equivalence can also be seen by comparing two ways to derive the quantum field equations. The first way, by which we derived (2), is to utilize the fact that the functional integral of functional derivative vanishes identically:

$$
\begin{aligned}
0 & =\frac{1}{N} \int d \phi \frac{\delta}{\delta \phi}\left(F[\phi] e^{i I[\phi]}\right) \\
& =\left\langle\frac{\delta F}{\delta \phi}\right\rangle+i\left\langle F[\phi] \frac{\delta I}{\delta \phi}\right\rangle
\end{aligned}
$$

where $F[\phi]$ is an arbitrary polynomial functional. The second way, ${ }^{3}$ analogous to (15), is to consider a change of integration variables, $\phi \rightarrow \phi+\alpha F[\phi]$, with an infinitesimal parameter $\alpha$ in the vacuum functional:

$$
\begin{aligned}
0 & =\frac{1}{N} \int d(\phi+\alpha F[\phi]) e^{i I[\phi+\alpha F[\phi]]}-\frac{1}{N} \int d \phi e^{i I[\phi]} \\
& =\frac{1}{N} \int d \phi\left(1+\int d y \alpha \frac{\delta F}{\delta \phi}\right)\left\{1+i \int d y\left[\alpha F[\phi] \frac{\delta I}{\delta \phi}+\partial_{\mu}\left(\alpha F[\phi] \frac{\partial \mathcal{L}}{\partial \partial_{\mu} \phi}\right)\right]\right\} e^{i I[\phi]}-\frac{1}{N} \int d \phi e^{i I[\phi]} \\
& =\epsilon\left[\left\langle\frac{\delta F}{\delta \phi}\right\rangle+i\left\langle F[\phi] \frac{\delta I}{\delta \phi}\right\rangle\right)
\end{aligned}
$$

where $\alpha$ is localized, as we did in (16), in the last line. Then one realizes that the first term in the last line of (17), which arises as the Jacobian factor in (18), results in the anomaly on the RHS of (3) for $F[\phi]=\bar{\delta} \phi$. In other words, Fujikawa's anomalous Jacobian is an integral form of the discrepancy between the classical and quantum field equations. This again proves the equivalence stated previously.

Our approach differs from Fujikawa's by the way the regularization is introduced. We have introduced it by defining the functional derivative in (8), not in the course of evaluating the Jacobian factor as he did. However, since the functional derivative should be defined in accordance with the path-integral measure to derive the field equations consistently, both regularization procedures should also be related to each other. As a consequence, the above equivalence may remain true after the regularization.

Our origin of anomalies, the RHS of (3), can be interpreted along the line of the conventional viewpoint in the operator formalism. ${ }^{7}$ Let us illustrate it for the case of the non-Abelian anomaly. The essential ingredient which causes the anomaly is the ill-defined term, $\langle\bar{\psi}(\delta I / \delta \bar{\psi})\rangle$ [or $\langle(\delta I / \delta \psi) \psi\rangle]$, that appears in (12). Noticing that, in the path-integral formalism, derivatives always operate outside the $T$ product, we may recast the term into

$$
\begin{aligned}
\left\langle\bar{\psi}(x) \frac{\delta I}{\delta \bar{\psi}(y)}\right\rangle=\langle\bar{\psi}(x) i \not D \psi(y)\rangle & =\partial_{\mu}^{y}\left\langle 0\left|T \bar{\psi}(x) i \gamma^{\mu} \psi(y)\right| 0\right\rangle+\left\langle 0\left|T \bar{\psi}(x) i A(y) \frac{1}{2}\left(1-\gamma^{5}\right) \psi(y)\right| 0\right\rangle \\
& =-i\left\langle 0\left|\left\{\psi^{\dagger}(x), \psi(y)\right\}\right| 0\right\rangle \delta\left(x^{0}-y^{0}\right)+\langle 0|T \bar{\psi}(x) i \not D \psi(y)| 0\rangle \\
& =i \delta(x-y) .
\end{aligned}
$$

Therefore, the singularity in the limit $y \rightarrow x$ stems from the equal-time anticommutator in the operator formalism, and is intrinsic to local quantum field theories. Our approach simplifies the process of deriving (19) by exploiting the quantum field equation (2), and gives meaning to the term by introducing the regularization for the functional derivative.

\section{CONCLUSION}

We have seen that, in the path-integral formalism, the classical field equations disagree with the quantum field equations and, as a result, anomalies may arise. With a suitable regularization for the functional derivative, we get the same expressions as the anomalous Jacobian gives. The origin of anomalies is shown to be equivalent to Fujikawa's anomalous Jacobian, and is also related to the conventional interpretation in the operator formalism. Remarkably, by the direct use of Noether's argument, anomalies manifest themselves as a breakdown of the current conservation associated with global symmetries, as expected. This is not the case for the anomalous Jacobian which is trivial for global transformations.

Although we have considered only linear transformations in this paper, our approach will be valid for more general (nonlinear) cases because the regularized functional derivative (8) satisfies the usual Leibniz law. Further, it is also valid when symmetries are expressed by invariances of the action, not of the Lagrangian. In this case, the Lagrangian changes in (1) by a total divergence of a term, which is accordingly absorbed into the Noether current. Thus the classical field equations are still crucial for the current to be conserved, and no essential change is needed for our argument. 
Interestingly, our approach is applicable even to the conformal anomaly in string theory, where the trace part of the classical energy-momentum tensor vanishes trivially without using any field equations. In this theory, we may redefine the string coordinates so as to preserve the reparametrization invariance in the path integral. This redefinition modifies the classical energy-momentum tensor, ${ }^{2,6}$ and the modified part gives a similar expression as the RHS of (3), which leads to the conformal anomaly.

\section{ACKNOWLEDGMENTS}

I would like to thank Professor G. Kramer for hospitality at the II. Institut für Theoretische Physik der Universität Hamburg. I also thank him for a careful reading of the manuscript and helpful comments. I am grateful to T. Berger, D. Dalmazi, and S.-L. Nyeo for enlightening discussions. This work was supported by funds provided by the Alexander von Humboldt Foundation.
${ }^{1}$ K. Fujikawa, Phys. Rev. Lett. 42, 1195 (1979); Phys. Rev. D 21, 2848 (1980); 22, 1499(E) (1980).

${ }^{2}$ For a review, see $\mathrm{K}$. Fujikawa, in Quantum Gravity and Cosmology, edited by H. Sato and T. Inami (World Scientific, Singapore, 1986).

${ }^{3}$ J. Zinn-Justin, Trends in Elementary Particle Theory, edited by H. Rollnik and K. Dietz (Lecture Notes in Physics, Vol. 37) (Springer, Berlin, 1975).

${ }^{4}$ S. D. Joglekar and A. Misra, Phys. Rev. D 36, 642 (1987).
${ }^{5}$ L. Alvarez-Gaumé and P. Ginsparg, Nucl. Phys. B243, 449 (1984).

${ }^{6}$ Equation (16) has been obtained for the conformal anomaly by K. Fujikawa, Phys. Rev. Lett. 44, 1733 (1980); Phys. Rev. D 23, 2262 (1981).

${ }^{7}$ See, e.g., S. Treiman, R. Jackiw, B. Zumino, and E. Witten, Current Algebra and Anomalies (World Scientific, Singapore, 1985). 